\title{
Neurocirurgia e filosofia no século XIX: como o pensamento da época influenciou $o$ desenvolvimento da neurocirurgia moderna
}

\section{Neurosurgery and Philosophy in the $19^{\text {th }}$ Century: How Modern Neurosurgery Development Relates to Past Thinking}

\author{
Flávio Ramalho Romero ${ }^{1}$ \\ ${ }^{1}$ Neurocirurgião, Hospital das Clínicas, Universidade Estadual de São \\ Paulo (Unesp), Botucatu, SP, Brasil \\ Arq Bras Neurocir 2015;34:258-262.
}

\begin{abstract}
Address for correspondence Flávio Ramalho Romero, MD, MSc, Departamento de Neurologia, Psiquiatria e Psicologia, Distrito de Rubião Júnior, s/n, Botucatu, SP, Brasil CEP: 18618-970 (e-mail: frromero@ig.com.br; romeroncr@gmail.com).
\end{abstract}

Divisão de Neurocirurgia, Escola de Medicina de Botucatu, Universidade Estadual de São Paulo (Unesp), Botucatu, SP, Brasil.

\section{Resumo \\ Palavras-Chave \\ - filosofia \\ - século XIX \\ - neurocirurgia moderna \\ - localização cerebral}

O século XIX foi um período marcado por profundas mudanças sociais, políticas e econômicas que refletiram diretamente no desenvolvimento científico. Neste artigo, tenta-se estabelecer relação entre as linhas filosóficas deste período e o surgimento da neurocirurgia moderna.

The 19th century was a period with important social, politic and economic changes that strongly supported scientific development. In this paper, I tried to establish relationship between philosophical theories of this period and beginning of modern neurosurgery.

\section{Introdução}

O século XIX foi um período marcado por importantes mudanças mundiais nos cenários político, econômico e social. ${ }^{1}$ Depois da queda do Império Francês e seus aliados nas guerras napoleônicas, o Império Britânico tornou-se a principal potência política e econômica mundial, sendo o berço da Revolução Industrial. Além disso, foi uma era de invenções e descobertas, com significante desenvolvimento - nos campos da matemática, física, química, biologia, elétrica e metalurgia - que lançou as bases para os avanços tecnológicos do século XX. As revoluções burguesas promoveram uma nova fase da ciência baseada na metodologia e no empirismo, desligando-a do caráter místico e artesanal dos séculos anteriores e oprimindo velhos cânones éticos do absolutismo e catolicismo. ${ }^{1-3}$

No campo das ciências médicas, o século XIX viu nascer a medicina experimental de Claude Bernard, ${ }^{4}$ a teoria de omnis cellula e cellula de Rudolf Virchow, ${ }^{5}$ a teoria da evolução das espécies de Charles Darwin ${ }^{6}$ e a genética de Gregor Mendel. ${ }^{7}$ 0 desenvolvimento da medicina se relacionou diretamente com a migração, superlotação das cidades e precárias received

August 18, 2014

accepted

June 12, 2015
DOI http://dx.doi.org/ $10.1055 / \mathrm{s}-0035-1559894$. ISSN $0103-5355$.
Copyright $(2015$ by Thieme Publicações License terms Ltda, Rio de Janeiro, Brazil
(1) (1) $\Theta \circledast$ 
condições de vida dos operários da Revolução Industrial. ${ }^{8}$ Como consequência, houve a proliferação de doenças infecciosas (tuberculose e sífilis) ou relacionadas à má alimentação (pelagra, escorbuto e raquitismo). Tais problemas são cruciais para entender a origem da medicina social de Rudolf Virchow $^{5}$ e as teorias sanitaristas de Edwin Chadwick. A mesma Revolução Industrial, junto com numerosas guerras e revoluções, gerou um desenvolvimento científico generalizado que contribuiu com a instauração de condições técnicas para o triunfo da assepsia, anestesia e cirurgia. ${ }^{9-11}$

O conhecimento da anatomia humana e a prevenção de doenças que ocorreram no século XIX foram responsáveis pela rápida aceleração do crescimento populacional no ocidente. A população europeia dobrou durante o século XIX, passando de cerca de 200 milhões de habitantes para mais de 400 milhões. Além disso, houve grande progresso nos meios de transporte, como a construção de ferrovias, que melhorou o modo de vida das pessoas e favoreceu os grandes movimentos de urbanização nos países ao redor do globo. ${ }^{8}$

Neste mesmo período, John Hunter aplicou o método experimental da ciência à cirurgia, dando nascimento à moderna cirurgia. ${ }^{12}$ Nas últimas duas décadas do século XIX, houve avanço da cirurgia em geral, mas especialmente

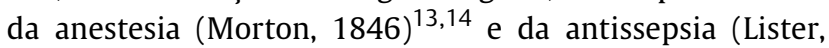
1867). ${ }^{15}$ O melhor conhecimento da anatomia e da função do sistema nervoso, com a teoria das localizações cerebrais (Broca, 1861), ${ }^{16-19}$ possibilitou o nascimento da neurocirurgia como especialidade médica moderna, graças, principalmente, aos pioneiros Victor Horsley e Harvey Cushing. ${ }^{20,21}$

\section{Filosofia no Século XIX}

O último terço do século XVIII, tempo do Iluminismo, produziu uma série de obras que desafiaram a filosofia desenvolvida até então. Immanuel Kant e Jean-Jacques Rousseau foram as figuras mais importantes desse período. A grande maioria dos filósofos do século XIX desenvolveu suas ideias sob forte influência das obras de Kant, explorando em particular o idealismo e a ética, que exibe uma tradição filosófica baseada na vontade-desejo do Ser Humano. Enquanto antes de Kant os filósofos tinham analisado, explorado e desenvolvido os objetos do conhecimento, os filósofos integrados na via idealística kantiana enveredaram na matéria do conhecimento, desenvolvendo ideias como as do ego e do eu, a mente e a consciência humana. ${ }^{22,23}$

Sigmund Freud não só constantemente recorreu a Kant, como aplicou e desenvolveu suas teorias sob forte influência kantiana. No caso Schreber (1911), Freud reporta-se ao trabalho de Kant - o modelo que encontrou na Crítica da razão pura ${ }^{22}$-observando que ambos têm ideias convergentes e modelos tripartidos. Enquanto Kant apresenta os elementos razão, entendimento e sensibilidade, Freud trabalha os conceitos de superego, ego e id. Tanto Kant como Freud afirmam que "algo" (alguma coisa) pode ser ou tornar-se consciente somente se for descrito, captado através da linguagem. ${ }^{24}$

Porém, em resposta a estes pensamentos, surgiram inúmeros absolutistas como Johann Gottlieb Fichte, Friedrich
Wilhelm Joseph von Schelling e Ralph Waldo Emerson, cujas ideias formaram a base do movimento conhecido como Romancismo, que buscou combinar a racionalidade formal do passado com um maior e mais imediato senso emocional e orgânico do mundo. O primeiro transformou o idealismo crítico de Kant em um idealismo absoluto, transformando o idealismo como última e máxima realidade. Para Fichte, o mundo foi criado por um ego absoluto, que no início apenas tinha consciência de si próprio. À medida que a criação se desenvolvia, tomava conhecimento do não eu, podendo distinguir as variedades contidas no mundo. Para ele, a vontade humana não é mais do que uma manifestação parcial do "eu" que concede aos seres a liberdade de agir. Ideias fundamentais que reluzem esta mudança apresentam-se na Evolução, como postulada por Goethe, Erasmus Darwin e Charles Darwin. ${ }^{25-27}$

Por outro lado, o filósofo alemão George Wilhelm Friedrich Hegel desenvolveu um sistema metafísico racionalista, historicista e absolutista, baseado na doutrina de que "o pensamento e o ser são o mesmo", e de que a natureza é a manifestação de um "Espírito Absoluto". Sob forte influência de Kant e Schelling, Hegel desenvolveu um sistema de idealismo absoluto, criando uma nova concepção da lógica e dos métodos filosóficos. A distinção de Hegel entre o irreconhecível e o desconhecido pode ser vista como o alicerce do seu sistema racional do universo. ${ }^{28-31}$

Este filósofo acreditava que a verdade absoluta, ou realidade, não só existe, como também que a mente humana é altamente capacitada para entender tudo através do conhecimento, porque "tudo o que é real é racional". Com base nestas ideias, Hegel desenvolveu um método em que as ideias em conflito se resolvem através de tríades em vários níveis. Cada tríade envolve a Tese (ideia ou movimento formador do primeiro estágio), a Antítese (opositora ao estágio anterior) e a Síntese (combina os elementos dos dois primeiros estágios opostos e permite concluir um novo e superior arranjo). Desta forma, a Síntese torna-se a Tese da tríade seguinte, e assim por diante. ${ }^{28-31}$

Este processo sem fim permite progredir até o Ideal, e dado a profundidade deste sistema dialético, o filósofo apela para que esse sistema deva ser aplicado a todos os campos do conhecimento, tanto na ciência como nas letras e nas artes. ${ }^{30}$ Portanto, consideram-se estas ideias fundamentais para o desenvolvimento científico baseado no empirismo e na metodologia, contribuindo diretamente para o desenvolvimento das ciências médicas e, consequentemente, da neurocirurgia moderna.

$\mathrm{Na}$ época a que nos reportamos, as discussões históricas de Hegel possuíam uma força inegável porque continham como contraforte a filosofia política e social mais tarde desenvolvida por Karl Marx (1818-1883), pensador revolucionário alemão que, juntamente com Friedrich Engels, fundou a base do comunismo moderno. ${ }^{32,33}$

Outras importantes mentes do século XIX que merecem destaque são ${ }^{34-36}$ :

- Arthur Schopenhauer (1788-1860) - idealista alemão que atribuiu à vontade um lugar de destaque em sua 
metafísica. Principal expoente do pessimismo; rejeitava o idealismo absoluto e pregava que a única atitude sustentável está na completa indiferença a um mundo irracional. Afirmava que o ideal maior é a negação do querer-viver.

- Auguste Comte (1798-1857) - francês; fundador do positivismo, um sistema que negava a metafísica transcendente e afirmava que a "divindade e o homem são um só", que o altruísmo é o dever maior do homem e que os princípios científicos explicam todos os fenômenos.

- Ludwig Feuerbach (1804-1872) - alemão; argumentava que a religião é uma projeção da natureza humana. Influenciou Marx.

- John Stuart Mill (1806-1873) - expoente inglês do utilitarismo; distinguia-se de Bentham ao reconhecer diferenças na qualidade e quantidade de prazer. Sobre $a$ liberdade (1859) é seu mais famoso trabalho.

- Soren Kierkgaard (1813-1855) - existencialista religioso dinamarquês cujo pensamento é a base do existencialismo moderno (ateísta). Pregava que a realidade residia apenas na existência e que o indivíduo possuía um valor singular.

- Herbert Spencer (1820-1903) - evolucionista inglês; sua "filosofia sintética" interpretava todos os fenômenos de acordo com o princípio do progresso evolucionário. (O ser humano teria que evoluir e respeitar as leis dentro de uma sociedade, e de acordo com a necessidade exigida pela maior parte dessa sociedade.)

- Charles S. Peirce (1839-1914) - físico e matemático norteamericano, fundador da escola filosófica chamada de "pragmatismo". Considerava a "lógica" a base da filosofia e entendia que o critério de uma crença é dado por suas consequências práticas.

- William James (1842-1910) - psicólogo e pragmatista norte-americano; afirmava que a realidade está sempre no "fazer-se" e que cada um deveria escolher a filosofia mais adequada a si próprio.

- Friedrich Wilhelm Nietzsche (1844-1900) - alemão; afirmava que a "vontade de poder" é básica na vida e que o espontâneo é preferível ao metódico. Atacou o "cristianismo", principalmente, por ser um sistema que apoiava os fracos, enquanto o valor maior pertence ao "além-dohomem". Foi um extraordinário poeta e romancista e é um dos mais influentes filósofos modernos. Por motivos de saúde, renunciou a um cargo em uma universidade na Suíça, em 1879, e passou a década seguinte escrevendo suas principais obras, no ritmo de um livro por ano. Sua existência criativa terminou em um colapso mental em 1889. Após sua morte, em 1900, sua irmã Elizabeth Foerster deliberadamente desvirtuou seus pensamentos com objetivos nacionalistas e antissemitas.

\section{O Surgimento da Neurocirurgia Moderna}

A trepanação é uma prática ancestral que remonta à Idade da Pedra. A razão pela qual o homem pré-histórico a realizava sempre foi tema de grandes debates. Alguns pesquisadores acreditam que fazia parte de rituais religiosos; outros defendem sua finalidade médica, como forma primitiva da neurocirurgia que conhecemos hoje. ${ }^{37,38}$ Porém, somente durante o século XIX foi possível o desenvolvimento de subsídios que sustentaram o nascimento da neurocirurgia moderna. O pensamento científico baseado na metodologia e no empirismo, quando aplicado às ciências médicas, trouxe grandes avanços nas áreas de assepsia e anestesia, bem como no conhecimento anatômico do sistema nervoso. ${ }^{30}$

O primeiro relato histórico de intervenção cirúrgica com anestesia geral data de 1846, no anfiteatro cirúrgico do Massachusetts General Hospital, em Boston, pelo cirurgião John Collin Waren, que realizou a exérese de um tumor no pescoço do paciente Gilbert Abbot, utilizando éter etílico. ${ }^{13}$ Nos anos seguintes, novos agentes foram desenvolvidos e utilizados. Ao óxido nítrico e ao éter seguiu-se o clorofórmio, utilizado pela primeira vez em 1847 durante um trabalho de parto, pelo médico inglês James Simpson. Em 1930 foi introduzido o ciclopropano, e em 1956, o halotanos. ${ }^{13,14}$ A partir daí, houve grande avanço nas técnicas anestésicas e na neuroanestesia.

O conhecimento dos microrganismos e das técnicas de assepsia e antissepsia tiveram no século XIX seu apogeu. Os trabalhos de Semmelwells (1846), Lister (1865) e Pasteur (1870) trouxeram enorme contribuição no conhecimento de agentes causadores de processos infecciosos, que eram grande complicadores dos atos cirúrgicos até então. ${ }^{15} \mathrm{~A}$ partir daí, Neuber (1882) introduziu o avental cirúrgico; Hasteld (1889), a luva de borracha; Schimmelbush (1891), a padronização da assepsia das mãos; e Radecki (1896), o uso de máscaras durante o ato cirúrgico, contribuindo para a redução da taxa de infecções em pacientes cirúrgicos. ${ }^{39}$

No início do século XIX houve um crescente interesse pela localização das funções cerebrais. Neste momento, surgiu a frenologia, que é o estudo da estrutura do crânio, de modo a determinar o caráter das pessoas e a sua capacidade mental. Esta pseudociência baseia-se na hipótese de que as faculdades mentais estão localizadas em "órgãos" cerebrais na superfície do crânio, que podem ser detectados por sua inspeção visual. O físico vienense Franz-Joseph Gall afirmou existirem 26 "órgãos" na superfície do cérebro que afetam o contorno do crânio, incluindo um “órgão da morte”, presente em assassinos. Sem dúvida, a teoria da frenologia foi importante por sugerir uma base científica para esta possibilidade, mas era baseada em inferências falhas, e não no método científico. ${ }^{40,41}$ Gall foi expulso de Viena pelas autoridades políticas e religiosas e mudou-se para Paris, onde solicitou ingresso na Academia Francesa de Ciências. Episódio curioso foi a interferência de Napoleão Bonaparte junto à academia para estabelecer uma comissão científica que julgasse o pedido de Gall. Este, porém, não apresentou seu já conhecido polêmico trabalho sobre os "órgãos da mente", mas sim um estudo sobre anatomia do cérebro, que era de classe internacional. Desta forma, seu pedido de ingresso junto à academia foi negado. Porém, coube a Pierre Flourens, renomado neurofisiologista da época, testar em animais a teoria frenológica de Gall, começando a desenvolver uma linha de pesquisa nessa área. ${ }^{42}$

Impulsionados pelos experimentos pioneiros de Flourens, por volta de 1825 , pesquisadores da época desenvolveram 
técnicas de ablação cirúrgica seletiva de partes do cérebro de animais e estimulação elétrica do córtex de animais e seres humanos. A partir daí, houve grande avanço na localização das funções cerebrais. Os experimentos de Flourens levaram à conclusão que os hemisférios cerebrais seriam os responsáveis pelas funções cognitivas, que o cerebelo regularia e integraria os movimentos, e que o tronco cerebral controlaria funções vitais, como a circulação, a respiração e a estabilidade geral do organismo. Por outro lado, ele não foi capaz de achar - provavelmente porque as espécies de animais que usou têm córtex relativamente primitivo - regiões mais específicas para memória e cognição, o que o levou a acreditar que elas estariam representadas de uma forma difusa por todo o cérebro. Deste modo, Flourens deduziu que diferentes funções realmente podiam ser atribuídas a regiões particulares do cérebro, mas faltava ainda uma localização mais precisa. ${ }^{43-45}$

Porém, na metade do século XIX, a partir de observações clínicas em indivíduos com transtornos de linguagem feitas na França e na Alemanha, foi possível associar funções mentais superiores com uma localização específica no córtex cerebral. Além disso, novos experimentos com estimulação elétrica mais precisa da superfície do córtex em primatas e cães, realizadas na Inglaterra e na Alemanha, forneceram uma prova mais forte de que havia localização estrita da função no cérebro.

A abordagem clínica foi utilizada de forma pioneira na França por Pierre Paul Broca. ${ }^{16-18}$ Em um trabalho clássico, realizado por volta de 1860-1870, ele estudou o cérebro de vários pacientes afásicos, entre eles um indivíduo que pronunciava apenas a expressão "tan". Após a morte deste paciente, Broca evidenciou que seu cérebro tinha uma lesão, secundária a neurossífilis, delimitada a uma área cortical de um dos hemisférios cerebrais anteriores. Esta parte do cérebro se tornou conhecida como área de Broca e é responsável pelo controle da expressão motora da fala. ${ }^{18}$ Mais ou menos na mesma época, um neurologista alemão, Carl Wernicke, descobriu uma área similar no lobo temporal, que, quando lesada, levava a um déficit sensorial da linguagem, ou seja, o paciente era incapaz de reconhecer palavras faladas, mesmo quando tivesse sua audição intacta. Wernicke postulou que esta área era conectada por sistemas de fibras nervosas à área de Broca, formando assim um sistema complexo, responsável pela compreensão e expressão da linguagem falada. ${ }^{46-48}$

Seus estudos foram confirmados por muitos neurologistas, incluindo John Hughlings Jackson, prodigioso neurologista inglês, que foi capaz de confirmar a localização da afasia e sua lateralidade, e proporcionar uma integração conceitual impressionante da localização funcional do cérebro, através de sua teoria das hierarquias nervosas, que era baseada na observação de que o pensamento e a memória eram menos afetados por lesões do que as funções mais inferiores, como o controle da respiração e da circulação. ${ }^{49-51}$

A estimulação elétrica de áreas corticais de seres humanos anestesiados foi inicialmente realizada pelo médico italiano Luigi Rolando, ${ }^{52}$ técnica também utilizada pelo neurocirurgião alemão Fedor Krause, ${ }^{53}$ que estimulou eletricamente as circunvoluções motoras de pacientes anestesiados para a remoção de tumores. Seu mapeamento das áreas corticais motoras foi notavelmente preciso, e deu apoio às pesquisas mais modernas.

Na mesma época dos estudos de Broca e Wernicke, dois fisiologistas alemães, Gustav Fritsch e Eduard Hitzig, aprimoraram o conhecimento sobre a localização cerebral da função, estimulando pequenas regiões com eletricidade, na superfície do cérebro de cães acordados. Eles descobriram que a estimulação de algumas áreas causavam contrações musculares na cabeça e pescoço, enquanto a estimulação de áreas cerebrais distintas causavam contrações das pernas anteriores ou posteriores. 54

Um neurologista inglês, Sir David Ferrier, realizou importantes experimentos baseados no trabalho de Fritsch e Hitzig, causando um tremendo impacto em nosso conhecimento sobre o cérebro. Entre 1870 e 1875, ele estimulou eletricamente os giros corticais expostos de macacos e de cães, mapeando 15 áreas relacionadas às funções motoras. Posteriormente, ele removeu cirurgicamente alguns desses pontos, demonstrando a abolição da função motora correspondente. ${ }^{55}$

Ferrier fez uma previsão ousada, ao transferir, com boa acurácia, como esses pontos poderiam ser representados no córtex humano, e usou este conhecimento com sucesso, pela primeira vez, para orientar diagnósticos neurológicos e cirurgias de pacientes com lesões ou tumores corticais. Por exemplo, ele previu corretamente a localização de uma lesão cortical em um paciente que tinha paralisia dos dedos e do antebraço de um lado, e deu a indicação para que o cirurgião, Macewen, operasse o paciente e removesse o tumor com maior precisão e certeza de sucesso. ${ }^{55}$

No final do século XIX, o conceito de localização cerebral foi firmemente estabelecido nas neurociências. Juntamente com o desenvolvimento da anestesia e da assepsia, foi fundamental para que Harvey Cushing e Victor Horsley desenvolvessem seus trabalhos, impulsionando o surgimento da neurocirurgia moderna.

\section{Conclusão}

O século XIX foi uma época marcada por enormes transformações políticas, econômicas e sociais que trouxeram à tona a necessidade de novos pensamentos e novas ideias. 0 rompimento com estigmas religiosos e ideias absolutistas abriu espaço para a observação e o empirismo, possibilitando o surgimento da metodologia aplicada a diversas áreas do conhecimento, inclusive à ciência. Este ambiente foi responsável por grandes revoluções tecnológicas, incluindo neste contexto as ciências médicas e, consequentemente, a neurocirurgia.

\section{Referências}

1 Perry M. Western Civilization: Ideas, Politics, and Society. Florence, KY: Cengage Learning; 2008

2 Hodge C. Encyclopedia of the Age of Imperialism, 1800-1944. Westport: Greenwood Publishing Group; 2008

3 Lyons M. Napoleon Bonaparte and the Legacy of the French Revolution. New York: St. Martin's Press, Inc.; 1994 
4 Bernard C. Introduction of the study of experimental medicine. Greene HC, trans. New York: Collier; 1961 (1865).

5 Virchow RLK. Cellular pathology, 1859 special ed., 204-207. London: John Churchill; 1978

6 Darwin C. The origin of species by means of natural selection. London: John Murray; 1850

7 Mendel G. Versuche über Plflanzen-hybriden. Verhandlungen des naturforschenden Ver-eines in Brünn, Bd. IV für das Jahr 1865, Abhand-lungen, 3-47 (1866).

8 Ashton TS. The Industrial Revolution (1760-1830). Oxford University Press; 1948

9 Ballance SC. A glimpse into the history of the surgery of the brain. Lancet 1922;22:111-116

10 Horrax G. Neurosurgery: an historical sketch. Springfield: Charles C. Thomas; 1952:10-15

11 Greenblatt SH. A history of Neurosurgery. Park Ridge: American Association of Neurological Surgeons; 1997:3-9

12 Kobler J. The Reluctant Surgeon. A Biography of John Hunter, New York, Doubleday, 1960

13 Leake CD. The Cadenced Story of Anesthesia. Letheon 1947

14 Sykes W. Essays on the First Hundred Years of Anesthesia. Stanley; 1960

15 Lister J. Antiseptic Principle of the Practice of Surgery. London 1867

16 Broca P. Sur les rapports anatomiques des divers points de la surface du crâne et des diverses parties des hémisphères cérébraux. Bull Soc D'Anth 1861;2:340

17 Broca P. Perte de la parole, ramollissement chronique et destruction partielle du lobe antérieur gauche du cerveau. Bull Soc Anthropl Paris 1861;2:235-238

18 Broca P. Diagnostic d'un abcès situé au niveau de la région du langage; trépanation de cet abcès. Rev Antropol 1876;5:244-248

19 Schiller F. Paul Broca. Founder of french anthropology, explorer of the brain. Berkeley: University of California Press; 1979

20 Laws ER Jr. Neurosurgery's man of the century: Harvey Cushingthe man and his legacy. Neurosurgery 1999;45(5):977-982

21 Horsley V. Brain surgery in the stone age. BMJ 1887;1:582-587

22 Kant I. Critique of Pure Reason. New York: Dover; 2003 (1771).

23 Watson J. The philosophy of Kant explained. J. Maclehose 1908: 62-72

24 Philips A. Becoming Freud: The making of a Psichoanalyst. Yale University press; 2014

25 Bonney R. Absolutism: what's in a name? French History 1987; I:93-117

26 Bornhein G. Filosofia do romantismo. In: GUINSBURG, J. (Ed.). O romantismo. 2.ed. São Paulo: Perspectiva; 75-1111985

27 Guinsburg J. O romantismo. São Paulo: Perspectiva; 2002

28 Hegel GWF. Enciclopedia of Philosophical science. Oxford: Clarendon Press; 1971

29 Hegel GWF. Elements of Philosophy of rights. Cambrigde: Cambridge University Press; 1991

30 Hegel GWF. Science of Logic. London: G. Allen and Unwin; 1969
31 Hegel GWF. The Phenomenology of Spirit. London: Harper and Row; 1967

32 Marx K. O capital. Coleção Os economistas. São Paulo: Nova Cultural; 1988

33 Marx K, Engels F. Obras escolhidas. Volume 1. São Paulo AlfaÔmega, sd.

34 Russell B. História da filosofia ocidental. São Pauo: Ed. Nacional Codil; 1967

35 Maritan J. Elementos de filosofia- I. Introdução geral a a filosofia. Rio de Janeiro: Agir; 1970

36 Hirschberger J. História da filosofia contemporânea. São Paulo: Herder; 1968

37 Broca A, Maubrac P. Traité de chirurgie cérebrale. Paris: Masson; 1896

38 Broca P. La trépanation chez les Incas. Bull. Acad Med 1866; 32:866-871

39 Tubino P, Alves E. História da Cirurgia. 2009

40 Gall FJ, Spurzheim JG. Anatomie et physionomie du système nerveux en general et du cerveau en particulier. Paris: F. Schoell; 1810

41 Gall FJ, Spurzheim JG. Recherches sur le système nerveux en général, et sur celui du cerveau en particulier. Paris1809

42 Greenblatt SH. Phrenology in the science and culture of the 19th century. Neurosurgery 1995;37(4):790-804, discussion 804-805

43 Tizard B. Theories of brain localization from Flourens to Lashley Med Hist 1959;3(2):132-145

44 Flourens MJP. Recherches expérimentales sur les propriétés et les functions du système nerveux dans les animaux vertébrés. Paris JB Ballière, 1842

45 Flourens MJP. Note touchant l'action de l'éther sur les centres nerveux. CR Acad Sci Paris 1847;24:340-344

46 Wernicke C. Lehrbuch der Gehirnkrankheiten. Berlim1900

47 Wernicke C. Der aphasische Symptomenkomplex. Berlim1874

48 Wernicke C. L'aphasie. Berlim1878

49 Jackson JH. Notes on the physiology and pathology of the nervous system. Med Times Gaz 1868

50 Jackson JH. Note on lateral deviation of the eyes in hemiplegia and in certain epileptiform seizures. Lancet 1866;i:311-331

51 Jackson JH. Note on the comparison and contrast of regional palsy and spasm. Lancet 1867;i:295-297

52 Caputi F, Spaziante R, de Divitiis E, Nashold BS Jr. Luigi Rolando and his pioneering efforts to relate structure to function in the nervous system. J Neurosurg 1995;83(5):933-937

53 Behrend CM. Fedor Krause und die Neurochirurgie. Zbl Neurochir 1938:53-135

54 Fritsch G, Hitzig E. On the electrical excitability of the cerebrum (1870), trans. G. von Bonin, in: Some Papers on the Cerebral Cortex, 73-96 (Thomas, Springfield, 1960).

55 Ferrier D. Experimental researches in cerebral physiology and pathology. West Riding Lunatic Asylum Medical Report 1873; 3:30-96 